\title{
Type 1 diabetes in Africa: an immunogenetic study in the Amhara of North-West Ethiopia
}

\author{
Shitaye A. Balcha ${ }^{1}$ • Abayneh G. Demisse ${ }^{2}$ - Rajashree Mishra ${ }^{3,4,5} \cdot$ Tanwi Vartak $^{6}$ - Diana L. Cousminer ${ }^{3,5,7}$. \\ Kenyaita M. Hodge ${ }^{3,5} \cdot$ Benjamin F. Voight ${ }^{7,8} \cdot$ Kim Lorenz $^{7,8} \cdot$ Stanley Schwartz $^{9} \cdot$ Samuel T. Jerram $^{6} \cdot$ Arla Gamper $^{10}$. \\ Alice Holmes $^{11}$. Hannah F. Wilson ${ }^{12}$ • Alistair J. K. Williams ${ }^{12}$. Struan F. A. Grant ${ }^{3,5,7,13,14}$ • R. David Leslie $^{6}$. \\ David I. W. Phillips ${ }^{15}$. Elisabeth R. Trimble ${ }^{16}$ (D)
}

Received: 1 February 2020 / Accepted: 1 June 2020 / Published online: 23 July 2020

(C) The Author(s) 2020

\begin{abstract}
Aims/hypothesis We aimed to characterise the immunogenic background of insulin-dependent diabetes in a resource-poor rural African community. The study was initiated because reports of low autoantibody prevalence and phenotypic differences from European-origin cases with type 1 diabetes have raised doubts as to the role of autoimmunity in this and similar populations. Methods A study of consecutive, unselected cases of recently diagnosed, insulin-dependent diabetes $(n=236, \leq 35$ years $)$ and control participants $(n=200)$ was carried out in the ethnic Amhara of rural North-West Ethiopia. We assessed their demographic and socioeconomic characteristics, and measured non-fasting C-peptide, diabetes-associated autoantibodies and HLA-DRB1 alleles. Leveraging genome-wide genotyping, we performed both a principal component analysis and, given the relatively modest sample size, a provisional genome-wide association study. Type 1 diabetes genetic risk scores were calculated to compare their genetic background with known European type 1 diabetes determinants.

Results Patients presented with stunted growth and low BMI, and were insulin sensitive; only $15.3 \%$ had diabetes onset at $\leq 15$ years. C-peptide levels were low but not absent. With clinical diabetes onset at $\leq 15,16-25$ and $26-35$ years, $86.1 \%, 59.7 \%$ and $50.0 \%$ were autoantibody positive, respectively. Most had autoantibodies to GAD (GADA) as a single antibody; the prevalence of positivity for autoantibodies to IA-2 (IA-2A) and ZnT8 (ZnT8A) was low in all age groups. Principal component analysis showed that the Amhara genomes were distinct from modern European and other African genomes. HLA-DRB1*03:01 $(p=0.0014)$ and $H L A-D R B 1 * 04(p=0.0001)$ were positively associated with this form of diabetes, while $H L A-D R B 1 * 15$ was protective $(p<0.0001)$. The mean type 1 diabetes genetic risk score (derived from European data) was higher in patients than control participants $\left(p=1.60 \times 10^{-7}\right)$. Interestingly, despite the modest sample size, autoantibody-positive patients revealed evidence of association with SNPs in the well-characterised MHC region, already known to explain half of type 1 diabetes heritability in Europeans.

Conclusions/interpretation The majority of patients with insulin-dependent diabetes in rural North-West Ethiopia have the immunogenetic characteristics of autoimmune type 1 diabetes. Phenotypic differences between type 1 diabetes in rural NorthWest Ethiopia and the industrialised world remain unexplained.
\end{abstract}

Keywords Africa · Autoantibodies · Ethiopia - Genomes $\cdot$ HLA $\cdot$ Rural $\cdot$ Type 1 diabetes

Shitaye A Balcha, Abayneh G. Demisse, Rajashree Mishra, Tanwi Vartak and Diana L. Cousminer are equal first authors. Alistair J. K. Williams, Struan F. A. Grant, R. David Leslie, David I. W. Phillips and Elisabeth R. Trimble are joint senior authors.

Electronic supplementary material The online version of this article (https://doi.org/10.1007/s00125-020-05229-x) contains peer-reviewed but unedited supplementary material, which is available to authorised users.

Elisabeth R. Trimble e.trimble@qub.ac.uk

Extended author information available on the last page of the article
Abbreviations
GADA Autoantibodies to GAD
GRS Genetic risk score
GWAS Genome-wide association study
IA-2A Autoantibodies to IA-2
PC Principal component
ZnT8A Autoantibodies to ZnT8 


\section{Research in context}

\section{What is already known about this subject?}

- Little is known about the immunogenetic basis of type 1 diabetes in sub-Saharan Africa, a region of wide genetic diversity

- Therefore, reports of low autoantibody prevalence have raised questions about the extent of autoimmune involvement

\section{What is the key question?}

- What is the autoimmune contribution to insulin-dependent diabetes among the Amhara in North-West Ethiopia?

\section{What are the new findings?}

- The Amhara genome is distinct from modern Europeans and other African ethnic groups

- Amhara with recently diagnosed type 1 diabetes have a high prevalence of autoantibodies

- The Amhara have immunogenetic features of autoimmune diabetes, similar to those found in adults in Europeanorigin populations

How might this impact on clinical practice in the foreseeable future?

- Urbanisation and associated increases in type 2 diabetes have tended to dominate the recent narrative about diabetes in sub-Saharan Africa. However, an equally large challenge is posed by type 1 diabetes and its management in rural areas where the majority of the population live. Can a management strategy for type 1 diabetes be developed that is sustainable in the long term and accessible to the rural patient? An effective way to achieve service sustainability with good long term outcomes for type 1 diabetes, despite a limited number of highly trained professional staff, is through the more widespread development of de-centralised, integrated networks of rural and urban clinics

\section{Introduction}

Type 1 diabetes is poorly characterised in many low- and middle-income countries of sub-Saharan Africa; specifically, there has been uncertainty about whether its pathogenesis is similar to the classic form of the disease found in industrialised countries. Striking phenotypic differences from the classic form of type 1 diabetes have been reported from several locations in sub-Saharan Africa; these include a low incidence in the pre-pubertal years with an age-specific peak in the third decade [1, 2], strong associations with low socioeconomic status [3], skewing of sex ratios with male predominance in some settings [4] and reported low autoantibody prevalence, suggesting a diminished role for autoimmune mechanisms in its aetiology compared with classic type 1 diabetes [5-7].

Classic type 1 diabetes is an autoimmune disease resulting from the interaction between genetic susceptibility [8] and the environment. Many loci have been shown to confer risk, of which the HLA class II genes remain the most important. Although the vast genetic diversity of Africa is well known $[9,10]$, very little is known about how, or if, this alters the genetic risk for type 1 diabetes in this region. Non-genetic, environmental factors also contribute to the pathogenesis of type 1 diabetes, their important role being inferred from the low concordance rate for the disease in identical twins, often less than $50 \%$ [11]. Environmental factors range from a putative role of viral infections in pancreatic beta cell death, see the review by Op de Beeck and Eizirik [12], to nutritional factors that may interact with genetic susceptibilities to determine disease risk [13]. There is a significant body of experimental evidence showing that moderate to severe, life-long undernutrition starting in utero affects pancreatic development and function by, inter alia, silencing key growth and differentiation factors [14-16], and by having an effect on the immune system [17]. Ethiopia is an example of a country that has a long history of repeated famines, and approximately $40 \%$ of children have evidence of nutritional stunting [18]. In these communities, undernutrition starts in utero and continues throughout life. The widespread experience of undernutrition in some areas of sub-Saharan Africa, taken in conjunction with the low levels of islet-cell autoimmunity in many historic reports from Africa [5-7], has raised the possibility that insulin-dependent diabetes in these populations may have a nutritional origin. Those with the lowest BMIs $\left(15-16 \mathrm{~kg} / \mathrm{m}^{2}\right)$ were said to have 'protein-deficient pancreatic diabetes' [19], a type of malnutrition-related diabetes which has since been removed from WHO classifications. So, although there is agreement that environmental factors are important, it is not easy to prove their aetiological significance in type 1 diabetes, 
probably because genetic factors are stable and environmental factors change with time.

On account of uncertainties about the role of autoimmunity in the pathogenesis of type 1 diabetes in sub-Saharan Africa, we have carried out an immunogenetic investigation in a consecutive, unselected cohort of newly diagnosed insulindependent diabetic participants from a community in Ethiopia. The genetic objectives were to investigate the genome-wide underpinnings of type 1 diabetes and the leading European type 1 diabetes risk loci in this population. The study was carried out in a rural community, the Amhara of North-West Ethiopia, whose socioeconomic conditions are typical of many regions in sub-Saharan Africa.

\section{Methods}

\section{Setting}

The study was based in Gondar, Ethiopia, a university city $750 \mathrm{~km}$ north-west of the capital, Addis Ababa. Gondar has a central university hospital and ten stable satellite/peripheral health centres, which provide care for all patients with diabetes in a predominantly rural health zone comprising 2.6 million people. In order for the study to be representative of both the rural and urban populations, the region chosen had to have a stable healthcare infrastructure that was inclusive of the $90 \%$ of the population who live in rural areas. The entire diabetes service in this region has been developed and overseen by the same consultant physician for more than 30 years; thus, diagnosis and treatment at both rural and urban clinics have been carried out under the oversight of the same clinical team with the same treatment and management plan. Epidemiological studies of the diabetes care and outcomes in this region have been extensively described [1, 4, 20].

\section{Participants}

Patients were entered sequentially into this study without selection bias. All patients and control participants were from the Amhara, the second-largest ethnic group in Ethiopia (approximately $29 \%$ of the total Ethiopian population); their language is Amharic, a Semitic language in the Afroasian group of languages [21] and, phenotypically, the Amhara have many Caucasoid features. This report includes data from patients aged up to 35 years with insulin-requiring diabetes and attending the University of Gondar Diabetes and Paediatric Clinics and associated rural clinics. All patients presented with a high plasma glucose and a degree of metabolic decompensation; all complained of weight loss (or parents worried about a very sick child), polydipsia and polyuria; most of the children and a high percentage of adults $(50 \%)$ were ketoacidotic at presentation. All patients included in the study required insulin treatment continuously from first presentation. Control participants were hospital attendees selected in the same manner as previously [3]; eligibility criteria for control participants included no history of diabetes, normal random plasma glucose, aged up to 35 years and belonging to the Amhara ethnic group. Control samples were used only for autoantibody, HLA and genome investigations.

\section{Clinical characterisation}

After metabolic stabilisation, a questionnaire was verbally administered in Amharic to record details of the duration and treatment of their diabetes, and details of their education, occupation and socioeconomic circumstances; height and weight were measured as previously described [3]. BMI was calculated as weight $(\mathrm{kg})$ divided by height squared $\left(\mathrm{m}^{2}\right)$. Age and sexadjusted height and BMI $z$ scores were derived using WHO Anthro software (version 3.2.2; https://www.who.int/ growthref/tools/en/; accessed 15 June 2019) [22]. Whole body bioimpedance was measured using a Bodystat meter (Bodystat, Douglas, Isle of Man) and fat mass was calculated by the method of Kotler et al [23]. Venous blood samples for study purposes were obtained at a median of 2.5 months (IQR 1-7) from diagnosis using vacutainers, and plasma separation was carried out on site; samples were stored at $-70^{\circ} \mathrm{C}$, initially in Gondar and subsequently in the UK.

\section{Laboratory analyses}

Autoantibodies to GAD (GADA), IA-2 (IA-2A) and ZnT8 (ZnT8A) were measured by radiobinding assays as previously described [24, 25]. All samples found to be GADA-positive using full-length GAD65 were re-assayed using truncated $\mathrm{GAD}_{65}(96-585)$ radiolabel [26]; whereas 17 (8.5\%) control participants were positive for GADA in the full-length GAD assay, the number were reduced to four $(2.0 \%)$ in the truncated GAD assay. As only 6 of 137 patients found positive for GADA with full-length GAD were negative in the truncated GADA assay, results for truncated $\mathrm{GAD}_{65}(96-585)$ were used in subsequent analyses. C-peptide was measured by Roche 'ECLIA' C-peptide chemiluminescence assay on a Cobas 8000 E602 machine (Switzerland), with a minimal detection limit of $0.010 \mu \mathrm{g} / \mathrm{l}$.

\section{Genotyping and quality control}

After extraction of DNA, patients and control participants were genotyped on the Infinium OmniExpress Exome Beadchip platform (Illumina, San Diego, CA, USA) at the Children's Hospital of Philadelphia Center for Applied Genomics (Philadelphia, PA, USA). Quality control was performed using PLINK (v.1.90Beta4.5) [27], excluding individuals with discordant sex information, duplicate individuals 
and individuals with missing genotype $>5 \%$. SNPs with a call rate $<95 \%$, minor allele frequency $<1 \%$ and Hardy-Weinberg equilibrium $p<10^{-5}$ were removed $(708,143$ SNPs remained).

HLA $D R$ selected genotypes were measured by the method of Bunce et al [28]; due to the remaining limited volume of extracted DNA, 188 of 236 patients and 152 of 200 control participants were HLA typed.

We carried out a genome-wide association study (GWAS), a genetic risk score (GRS) analysis for type 1 diabetes and principal component (PC) analyses: details are in the Statistical analysis section (below).

\section{Statistical analysis}

Anthropometric, metabolic, autoantibody and HLA statuses of patients were tested by $t$ tests or ANOVA for continuously distributed variables (using log transformation where appropriate) or by $\chi^{2}$ tests for discrete variables. A $p$ value of $<0.05$ was considered to be statistically significant.

GWAS was carried out using a univariate linear mixed model within GEMMA (https://github.com/geneticsstatistics/GEMMA v. 0.94) [29], which accounts for population stratification and relatedness using the Wald test. Additionally, 55 established type 1 diabetes-implicated signals and their proxy SNPs were tested (11,748 SNPs) [30], and 403 established type 2 diabetes-implicated variants and their proxy SNPs were also tested (24,926 SNPs) [31].
Proxy SNPs were found using raggr (http://raggr.usc.edu, v. 3 . 5.0, accessed 26 July 2018), with a linkage disequilibrium threshold of $r^{2}<0.8$ in the European and African populations. The association tests were also performed in a restricted set of cases positive for at least one autoantibody.

GRS for type 1 diabetes was calculated using PLINK by multiplying the number of risk-increasing alleles by the natural $\log$ of the OR at each locus and summing the OR across risk loci for each individual. Included were 19 SNPs (electronic supplementary material [ESM] Table 1) in the GRS, using weights as previously described [32]. The distribution of the type 1 diabetes GRS was compared for all diabetes cases vs control participants and for autoantibody-positive cases using linear regression, adjusting for sex and the first four PCs.

PC analysis was performed using PLINK as follows: the 1000 Genomes [33] and Ethiopian genotype files were merged, removing 4277 SNPs with location conflicts. SNPs with a minor allele frequency $<0.01$ were removed, and linkage disequilibrium pruning was performed at $r^{2}<0.2$ between any two SNPs. Also removed was one individual from each pair with an identity-by-descent value $>0.3$ (46 individuals removed). PLINK was then used to calculate PCs and R (v.3.5.0) was used to plot the first three PCs.

\section{Ethical approval}

The study was approved by the institutional ethics review boards of Gondar College of Medicine and Health Sciences

Table 1 Metabolic characteristics and autoantibody status at presentation of Ethiopian patients with type 1 diabetes

\begin{tabular}{|c|c|c|c|c|c|}
\hline \multirow[t]{2}{*}{ Characteristic } & \multicolumn{4}{|l|}{ Age at onset (year) } & \multirow[t]{2}{*}{$p$ value } \\
\hline & $0-15$ & $16-25$ & $26-35$ & All & \\
\hline Number tested & 36 & 124 & 76 & 236 & \\
\hline Male, $n(\%)$ & $16(44.4)$ & $90(72.6)$ & $59(77.6)$ & $165(69.9)$ & 0.001 \\
\hline Blood glucose at diagnosis, mmol/l, median (IQR) & $29.3(27.1-33.3)$ & $29.7(24.0-33.3)$ & $26.1(20.4-32.2)$ & $28.3(22.2-33.3)$ & 0.03 \\
\hline Insulin dose after stabilisation, $\mathrm{U} / \mathrm{kg} /$ day, mean (SD) & $0.92(0.37)$ & $0.79(0.23)$ & $0.66(0.18)$ & $0.77(0.26)$ & $<0.001$ \\
\hline Diabetes duration, months, median (IQR) & $3(1-7)$ & $2(1-6)$ & $2(1-7)$ & $2.5(1-7)$ & NS \\
\hline C-peptide, $\mu \mathrm{g} / \mathrm{l}$, median (IQR) & $0.46(0.32-1.09)$ & $0.77(0.33-1.35)$ & $0.98(0.46-1.87)$ & $0.80(0.34-1.42)$ & 0.03 \\
\hline Height, SD $z$ score, mean (SD) ${ }^{\mathrm{a}}$ & $-1.49(1.09)$ & $-1.18(0.91)$ & $-1.09(0.83)$ & $-1.20(0.92)$ & NS \\
\hline BMI, SD $z$ score, mean $(\mathrm{SD})^{\mathrm{a}}$ & $-1.20(1.14)$ & $-1.44(1.11)$ & $-0.97(1.16)$ & $-1.25(1.15)$ & 0.02 \\
\hline \% body fat, mean (SD) & $6.2(8.7)$ & $11.9(7.7)$ & $13.7(6.5)$ & & $<0.001$ \\
\hline Rural birth, $n(\%)$ & $30(83.3)$ & $115(92.7)$ & $66(86.8)$ & $211(89.4)$ & NS \\
\hline \multicolumn{6}{|l|}{ Autoantibody prevalence, $n(\%)^{\mathrm{b}}$} \\
\hline GADA $^{\mathrm{c}}$ & $29(80.6)$ & $69(55.6)$ & $33(43.4)$ & $131(55.5)$ & 0.001 \\
\hline IA-2A & $1(2.8)$ & $5(4.0)$ & $2(2.6)$ & $8(3.4)$ & NS \\
\hline ZnT8A & $6(16.7)$ & $11(8.9)$ & $7(9.2)$ & $24(10.2)$ & NS \\
\hline Any antibody present & $31(86.1)$ & $74(59.7)$ & $38(50.0)$ & $143(60.6)$ & 0.001 \\
\hline
\end{tabular}

${ }^{\text {a }}$ Based on WHO standards, see Methods

${ }^{\mathrm{b}}$ Note: Non-diabetic controls, $n=200$ : positive for autoantibodies, GADA, $n=4$; IA-2A, $n=2$; ZnT8A, $n=5$; any antibody, $n=11$

${ }^{\mathrm{c}} \mathrm{GADA}$, antibodies against truncated GAD, i.e. $\operatorname{GAD}_{65}(96-585)$ 
and the UK National Research Ethics Services Committee (REC; reference: 14/WA/0132). Written, informed consent was obtained from all participants or their parents, as appropriate.

\section{Results}

The characteristics of the diabetes cases are shown in Table 1; results for antibody positivity in control participants are shown separately below the patient results. Patients were investigated at a median of 2.5 months after clinical diagnosis. The overwhelming majority (89.4\%) of the participants were born in rural areas around Gondar, Ethiopia. The median age at diagnosis was 21 years; only $36(15.3 \%)$ were diagnosed at 15 years of age or younger, and most cases were in the 1625 year age group. There was a striking male preponderance, but only in the post-pubertal age groups: $72.6 \%$ in the $16-$ 25 year age group and $77.6 \%$ in the $26-35$ year age group. The mean BMI SD $z$ scores were lower than WHO norms in all age groups; in the adult groups, the BMIs (mean [SD]) were equivalent to $18.6(2.4)$ and $19.8(2.9) \mathrm{kg} / \mathrm{m}^{2}$ in the $16-25$ and $26-35$ year age groups, respectively. The percentage of body fat was also low in all age groups. All cases had low, but detectable, non-fasting C-peptide levels. The insulin treatment doses were $<1 \mathrm{U} / \mathrm{kg} /$ day for all age groups. Of the 236 cases, $112(47.5 \%)$ reported that their families' source of income was subsistence farming or labouring, while only 41 (17.4\%) had paid employment or owned businesses. Educational levels were low, with only 74 of the 200 adults $(37 \%)$ reporting completed secondary education (not shown).

\section{Autoantibody status}

Table 1 shows that $86.1 \%$ of cases in the $0-15$ year age group, $59.7 \%$ of cases in the $16-25$ year age group and $50.0 \%$ of cases in the 26-35 year age group were autoantibody positive. GADA was the most common autoantibody and the most common when there was a single autoantibody; thus, 114 of 143 autoantibody-positive cases had GADA as the only autoantibody. Of control participants, $2 \%$ were GADA positive. The prevalence of GADA declined from $80.6 \%$ in those aged $\leq 15$ years to $55.6 \%$ in the $16-25$ year age group and $43.4 \%$ in the 26-35 year age group. In contrast, the prevalence rates of ZnT8A and IA-2A were low, even in those with childhood onset, without any age-specific trend. The GADA-positive and GADA-negative groups were further compared (ESM Table 2). In comparison with the autoantibody (GADA)-positive group, the negative (GADA) group were slightly older (23.6 vs 20.4 years, $p<0.001$ ), had lower plasma glucose at presentation ( 26.7 vs $29.8 \mathrm{mmol} / \mathrm{l}, p=0.01$ ), had higher Cpeptide levels ( 0.96 vs $0.71 \mu \mathrm{g} / \mathrm{l}, p=0.015)$ and had lower insulin requirements $(0.72 \mathrm{vs} 0.81 \mathrm{U} / \mathrm{kg}, p=0.012)$. They had similar BMIs (SD $z$ score -1.25 , or $19.3 \mathrm{~kg} / \mathrm{m}^{2}$ [mean for both]). Percentage body fat was not significantly different in the two groups: GADA negative, $12.7 \%$ vs GADA positive, $10.8 \% ; p=0.065$.

\section{Genomic analysis}

$\mathrm{PC}$ analysis $\mathrm{PC}$ analysis is a technique that identifies the major axes of variation in genetic data. Leveraging the genome-wide genotyping data, the first PCs were plotted against the 1000 Genomes reference set [33] to visualise the relationship of the Ethiopian samples against worldwide super-populations (Fig. 1a, b). Comparing PC1 with PC2 (Fig. 1a), a clear separation was observed of the Ethiopian sample from the 1000 Genomes groups, and plotting PC2 vs PC3 (Fig. 1b) revealed clearly that the sample from the Amhara of Ethiopia was distinct from European and other African populations.

HLA analysis $H L A-D R B 1 * 03: 01$ was positively associated with diabetes $(81 / 188,43.1 \%)$ compared with control participants $(40 / 152,26.3 \%$; OR 2.12; $p=0.0014)$, and this association persisted when considering only GADA-positive cases $(54 / 102,52.9 \%)$ (Table 2). HLA-DRB1*04 was also positively associated with diabetes $(83 / 188,44.1 \%)$ vs control participants $(37 / 152,24.3 \%$; OR $2.46 ; p=0.0001)$; this association persisted when GADA-positive cases only were considered $(51 / 102,50.0 \%)$. HLA-DRB $1 * 15$ was strongly protective in the total diabetes group $(7 / 188,3.7 \%)$ compared with control participants $(25 / 152,16.4 \%$; OR $0.20 ; p<0.0001)$; this association persisted when GADA-positive cases only were considered $(2 / 102,2.0 \%)$. In GADA-negative cases, these three HLA disease associations were less striking (HLA$D R B 1 * 03: 01[31.4 \%, p=0.40]$ ), but, importantly, with significant risk with $H L A-D R B 1 * 04(37.2 \%, p=0.035)$ and significant protection with $H L A-D R B 1 * 15(5.8 \%, p=0.018)$.

GRS GRS for type 1 diabetes is shown in Fig. 2, and the type 1 diabetes-associated SNPs used in the GRS analysis are shown in ESM Table 1.

The average type 1 diabetes GRS was significantly higher for diabetes cases than for control participants. The mean (SD), compared with that of the control participants of 0.154 (0.067), was 0.189 (0.064) for the total diabetes group, 0.199 $(0.067)$ for the autoantibody-positive group and $0.171(0.057)$ for the autoantibody-negative group, with $p=1.6 \times 10^{-7}, p=$ $1.54 \times 10^{-9}$ and $p=\mathrm{NS}$, respectively.

GWAS (ESM Figs 1, 2, 3, Table 3; ESM Tables 3, 4) No single SNP achieved genome-wide significance $\left(p<5 \times 10^{-8}\right)$ when all diabetic participants were investigated as a single group, irrespective of autoantibody positivity (ESM Figs 1 and 2). However, despite the modest sample size, SNPs within the HLA region were border-line GWAS significant $(p<5 \times$ 
Fig. 1 PC analysis. (a) The first two PCs (PCs 1 and 2). (b) The second and third PCs (PCs 2 and $3)$. These are based on genomewide genotypes of the Ethiopian (Amhara) participants compared with 1000 Genomes ancestral groups. AFR, African; AMR, Americas. EAS, East Asian. ETP, Ethiopian; EUR, European; SAS, Southeast Asian a

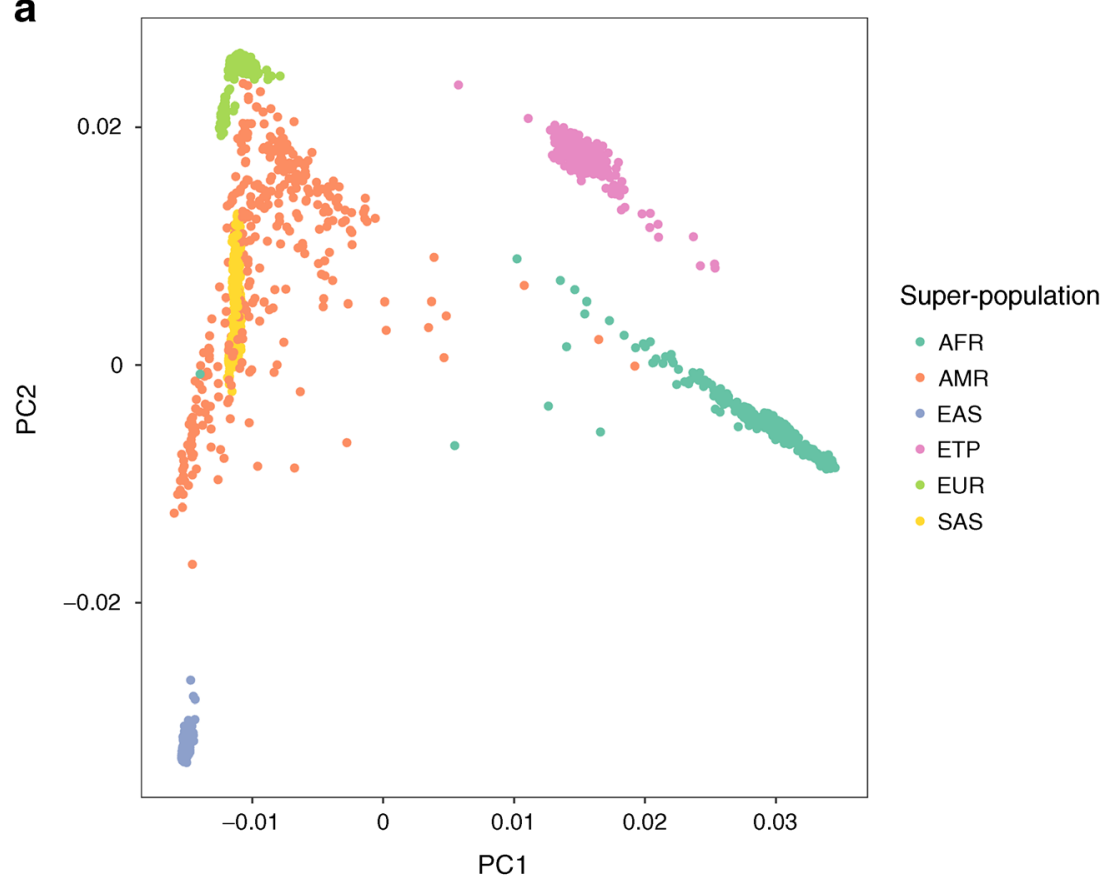

b

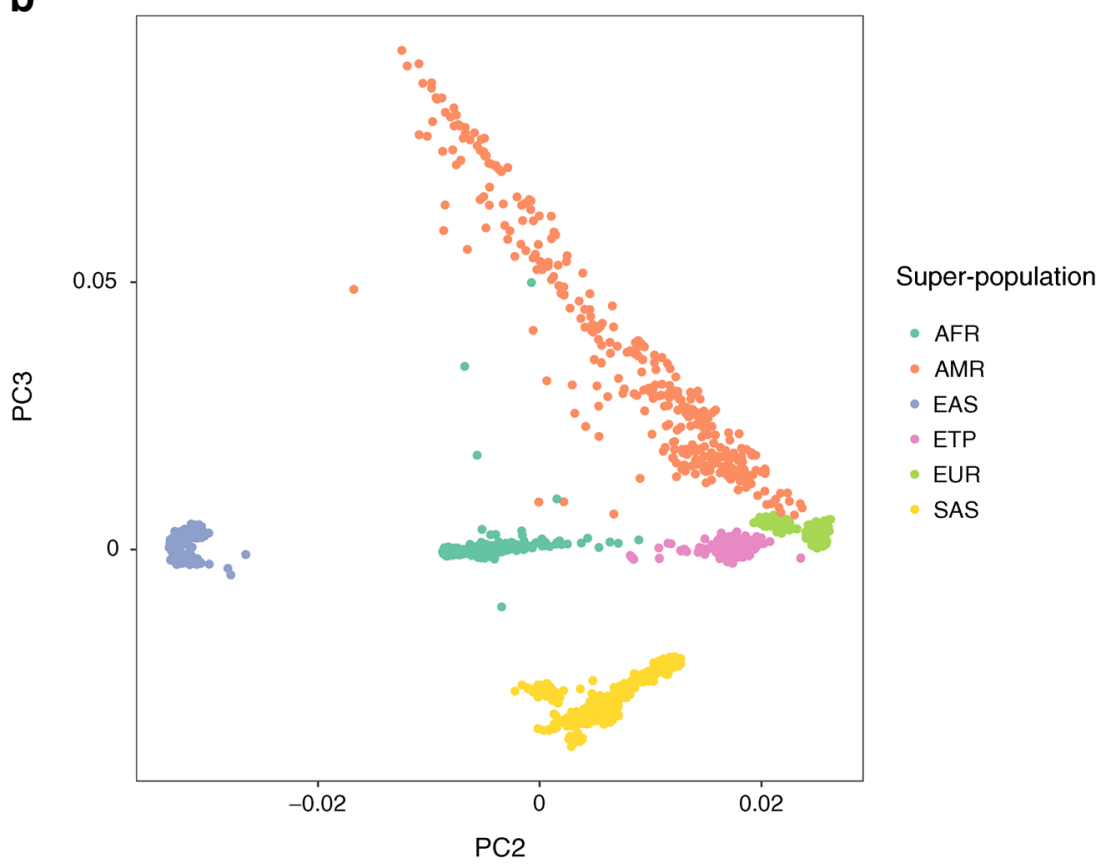

$10^{-6}$ ) for autoantibody-positive patients (Table 3, ESM Fig. 2 ), with the strongest signal falling in the $H L A-D Q B 1$ locus (rs9273363, $p=5.13 \times 10^{-8}$; Table 3 ). We also observed suggestively associated signals on chromosomes 4,16 and 3 (Table 3). The GWAS analysis did not show genomic inflation $(\lambda=1.01$; ESM Fig. 3$)$. We then took a candidate SNP approach and extracted all type 1 and type 2 diabetesassociated SNPs; the strongest type 1 diabetes-associated signal was in the HLA-DQB1 region (rs1063355, $p=6.28 \times$
$10^{-6}$ ) (ESM Table 3); however, no type 2 diabetes-associated loci achieved significance (ESM Table 4).

\section{Discussion}

This detailed study of consecutive, unselected, newly diagnosed patients in an impoverished, mainly rural population in sub-Saharan Africa shows that the majority have low C- 
Table 2 Analysis of the frequency of the three most prominent type 1 diabetes risk alleles in European-origin populations among Ethiopian patients with diabetes and non-diabetic controls

\begin{tabular}{|c|c|c|c|c|c|c|c|c|c|c|}
\hline \multirow[t]{2}{*}{ Allele } & \multicolumn{3}{|c|}{ Patients with diabetes, $n(\%)$} & \multirow{2}{*}{$\begin{array}{l}\text { Controls } \\
n=152\end{array}$} & \multicolumn{2}{|c|}{ All diabetes vs controls } & \multicolumn{2}{|c|}{$\begin{array}{l}\text { GADA-positive diabetes vs } \\
\text { controls }\end{array}$} & \multicolumn{2}{|c|}{$\begin{array}{l}\text { GADA-negative diabetes vs } \\
\text { controls }\end{array}$} \\
\hline & $\begin{array}{l}\text { All } \\
n=188\end{array}$ & $\begin{array}{l}\text { GADA } \\
\text { positive } \\
n=102\end{array}$ & $\begin{array}{l}\text { GADA } \\
\text { negative } \\
n=86\end{array}$ & & OR $(95 \% \mathrm{CI})$ & $p$ value & OR $(95 \% \mathrm{CI})$ & $p$ value & OR $(95 \% \mathrm{CI})$ & $p$ value \\
\hline$D R B 1 * 03: 01$ & $81(43.1)$ & $54(52.9)$ & $27(31.4)$ & $40(26.3)$ & $2.12(1.34-3.37)$ & 0.0014 & $3.15(1.85-5.35)$ & $<0.0001$ & $1.38(0.78-2.44)$ & 0.40 \\
\hline$D R B 1 * 04$ & $83(44.1)$ & $51(50.0)$ & $32(37.2)$ & $37(24.3)$ & $2.46(1.54-3.93)$ & 0.0001 & $3.11(1.82-5.32)$ & $<0.0001$ & $1.84(1.04-3.27)$ & 0.035 \\
\hline$D R B 1 * 15$ & $7(3.7)$ & $2(2.0)$ & $5(5.8)$ & $25(16.4)$ & $0.20(0.08-0.47)$ & $<0.0001$ & $0.10(0.02-0.43)$ & 0.0001 & $1.31(0.12-0.85)$ & 0.018 \\
\hline
\end{tabular}

peptide levels and low BMI, as well as diabetes-associated autoantibodies and diabetes risk alleles for type 1 diabetes; a smaller group are autoantibody negative and have some minor differences in their profile. As with previous studies in this and other locations in the region, the cases have a different disease phenotype; thus, the median age of onset is later than that observed in most industrialised countries and there is a striking male predominance in the post-pubertal age groups.

\section{Autoantibody prevalence}

The prevalence of diabetes-related autoantibodies was high, falling from $86.1 \%$ in the youngest age group to $50.0 \%$ in the oldest group. This autoantibody frequency and age dependence were similar to $[34,35]$, if slightly lower [36] than, some other reports from industrialised countries, with all

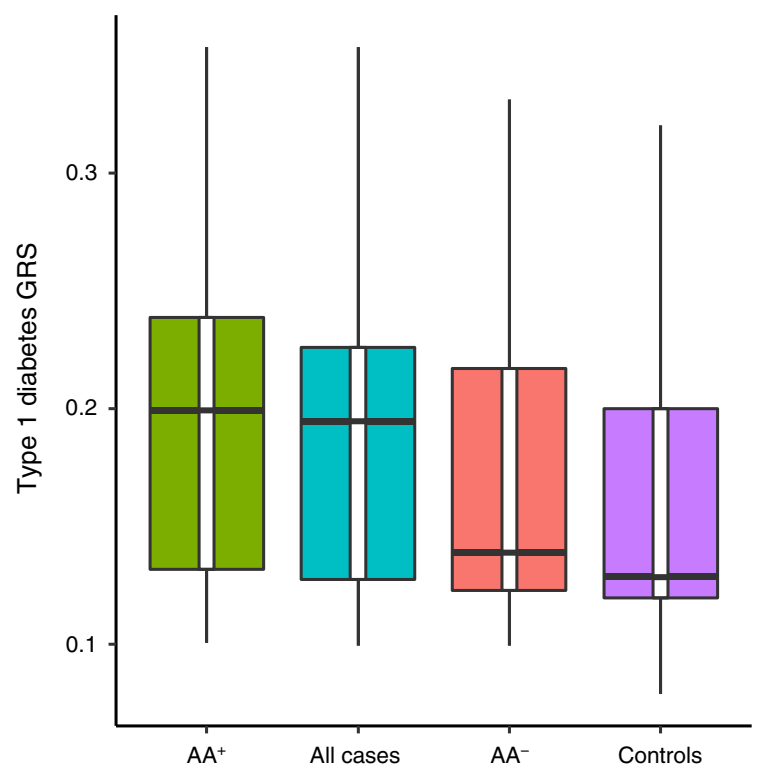

Fig. 2 Box and whisker plot showing GRS for type 1 diabetes. The GRS was calculated using 19 established type 1 diabetes-associated SNPs of European background; variants, risk alleles and weights are listed in ESM Table 1 (see the Methods/Statistical analysis section for details). Diabetic patients were compared with control participants: autoantibody-positive cases $\left(\mathrm{AA}^{+}\right)\left(n=121\right.$, vs controls, $\left.p=1.54 \times 10^{-9}\right)$, all cases $(n=187$, vs controls, $\left.p=1.6 \times 10^{-7}\right)$, autoantibody-negative cases $\left(\mathrm{AA}^{-}\right)(n=66$, vs controls, $p=\mathrm{NS})$ and control participants $(n=137)$. The vertical lines denote the maximum and minimum values demonstrating lower prevalence of autoantibody positivity with increasing age of onset. However, whereas the majority of type 1 diabetes patients in industrialised countries have multiple diabetes-related autoantibodies, in this present Ethiopian study most had GADA alone, which was evident even in the $0-15$ age group. Of note, the prevalence of autoantibodies to full-length GAD was relatively high in control participants, at $8.5 \%$ (mentioned in Methods section); however, in contrast to cases, the majority of these autoantibodies were directed to the low-risk N-terminal epitope [26]. The prevalence of other autoantibodies, IA-2A and ZnT8A, was low in all age groups, including those with childhood-onset disease. Our findings contrast with two urban studies from Ethiopia that found much lower levels of GADA, albeit with very low or absent IA-2A; however, these participants were studied 6 or more years after diagnosis [7, 37]. Unfortunately, comparisons in sub-Saharan Africa are difficult because there are very few studies where blood sampling for autoantibody status has taken place close to the time of diagnosis and involved both urban and rural populations, with the rural population forming the majority in most countries in this region. Moreover, the vast genetic diversity in this continent also complicates comparisons $[9,10,38]$. A recent study of type 1 diabetes from West Africa (Cameroon) investigated an urban group shortly after clinical diagnosis and compared them with a Belgian population. As in Ethiopia, few (9\%) had childhood-onset type 1 diabetes, and in those who were classified as having type 1 diabetes a very low percentage of children ( $\leq 15$ years) and adults (median age 30 years) were autoantibody positive; the majority of these had GADA, and far fewer had IA-2A or ZnT8A [2]: the overall autoantibody prevalence in those classified as having type 1 diabetes was lower than in the Amhara. The adult Belgian diabetic participants had a much higher incidence of multiple autoantibodies than the Cameroonian participants. The West Africans of Cameroon are of Bantu background and belong to a different genomic group to the Amhara [9, 10,38]. These findings of different autoantibody profiles among ethnic groups are echoed by studies in South Africa comparing the profiles of type 1 diabetes in black (mixed ethnicity) and white participants. Interestingly, the peak age of clinical onset was later in 
Table 3 GWAS: border-line genome-wide significant signals associated with autoantibody-positive diabetes cases $\left(p<5 \times 10^{-6}\right)$

\begin{tabular}{|c|c|c|c|c|c|c|c|c|c|}
\hline SNP & Chromosome & Position $^{\mathrm{a}}$ & Minor/major allele & $\mathrm{MAF}^{\mathrm{b}}$ in cases & MAF in controls & OR & CI & $p$ & Locus \\
\hline rs 9273363 & 6 & 32626272 & $\mathrm{~A} / \mathrm{C}$ & 0.445 & 0.217 & 1.281 & $1.226-1.339$ & $5.13 \times 10^{-8}$ & $H L A-D Q B 1$ \\
\hline rs2760985 & 6 & 32566398 & $\mathrm{~A} / \mathrm{G}$ & 0.261 & 0.088 & 1.363 & $1.288-1.443$ & $1.16 \times 10^{-7}$ & $H L A-D R B 1$ \\
\hline rs9268528 & 6 & 32383108 & $\mathrm{G} / \mathrm{A}$ & 0.407 & 0.358 & 1.261 & $1.208-1.316$ & $1.57 \times 10^{-7}$ & BTNL2 \\
\hline rs9268542 & 6 & 32384721 & $\mathrm{G} / \mathrm{A}$ & 0.407 & 0.365 & 1.256 & $1.203-1.312$ & $3.23 \times 10^{-7}$ & BTNL2 \\
\hline rs 11947273 & 4 & 92544404 & $\mathrm{~T} / \mathrm{C}$ & 0.336 & 0.445 & 0.811 & $0.778-0.846$ & $1.25 \times 10^{-6}$ & CCSER1 \\
\hline rs2187818 & 6 & 32395568 & $\mathrm{G} / \mathrm{T}$ & 0.360 & 0.427 & 0.805 & $0.770-0.841$ & $1.48 \times 10^{-6}$ & $H L A-D R A$ \\
\hline rs9268585 & 6 & 32397403 & $\mathrm{~T} / \mathrm{G}$ & 0.360 & 0.427 & 0.805 & $0.770-0.841$ & $1.48 \times 10^{-6}$ & $H L A-D R A$ \\
\hline rs4784939 & 16 & 58468211 & $\mathrm{~T} / \mathrm{C}$ & 0.309 & 0.146 & 1.280 & $1.216-1.347$ & $2.43 \times 10^{-6}$ & GINS3 \\
\hline rs996482 & 3 & 20583674 & $\mathrm{~T} / \mathrm{C}$ & 0.318 & 0.172 & 0.817 & $0.782-0.853$ & $4.06 \times 10^{-6}$ & SGOL1 \\
\hline
\end{tabular}

${ }^{a}$ Base pair position reported for genome build 37

${ }^{\mathrm{b}} \mathrm{MAF}$, minor allele frequency. Note that minor allele is the effect allele

Statistics: Linear mixed model with Wald test in GEMMA

black compared with white participants, and both groups were heavier than the Amhara (average BMI for black and white participants, $24.0 \mathrm{~kg} / \mathrm{m}^{2}$ and $22.4 \mathrm{~kg} / \mathrm{m}^{2}$, respectively). When studied several years after clinical diagnosis (mean duration $>5$ years), overall GADA positivity was $60 \%$ and $66 \%$ for black and white participants, respectively; IA-2A positivity was lower in the former than the latter (19\% vs $41 \%$, respectively), especially in those aged $>21$ years at diagnosis $(7.3 \%$ vs 33\%, respectively) [39]. Taken together with our data, these results $[2,39]$ from three different regions of sub-Saharan Africa suggest that there is considerable heterogeneity in the prevalence of autoantibody profiles both within Africa and between African and European populations. These differences appear to be independent of the age of onset of diabetes and, for the present, remain unexplained.

\section{Genomic analysis}

Using genome-wide genotyping data, our study population was shown to be distinct from European and other African ancestral groups; based on what is known of their demographic and linguistic history, this was not unexpected [10]. GWAS and HLA analysis confirmed the predominant HLA association with type 1 diabetes-associated risk and protective alleles. By contrast, and of note, no associations were found for type 2 diabetes-associated loci. However, our sample size was low and power limited, and therefore the results should be seen as relatively preliminary. In view of the autoantibody and HLA results a GRS was applied, based on SNPs associated with type 1 diabetes on a European genetic background. This was done with the knowledge that the results might not be as robust when applied to a different ethnic group [40]. Despite the European background of the SNPs employed, the Ethiopian diabetes group showed a significantly increased GRS compared with control participants, mainly associated with autoantibody-positive cases. In addition, these Amhara diabetes patients showed an association with HLA class II alleles $H L A-D R B 1^{*} 03: 01$ and $H L A-D R B 1 * 04$, in both the total diabetes cohort as well as in those with GADA, while $H L A-D R B 1 * 15$ was strongly protective for diabetes. GADAnegative patients demonstrated an association with HLA$D R B 1 * 04$ and protection with $H L A-D R B 1 * 15$. In short, they have some of the immunogenic features of the autoimmune diabetes of adolescent and adult European-origin and Chinese populations [41], while the diabetes-associated autoantibody, GADA, was similarly the dominant autoantibody and associated with $H L A-D R B 1 * 03: 01$, consistent with heterogeneity of type 1 diabetes endotypes $[42,43]$. Our observations imply a widespread commonality in GADA-dominant, HLAassociated adult-onset autoimmune diabetes, despite global variation in the precise HLA-associated genotypes. Moreover, as with adult-onset autoimmune diabetes in Europe and China, both IA-2A and ZnT8A had lower frequencies and, in the present Amhara cohort, even in childhood-onset type 1 diabetes the prevalence of IA-2A was very low.

\section{Implications}

Given that the majority of patients with diabetes in this rural Ethiopian population appear to have an autoimmune basis to their diabetes, the results do not support the hypothesis previously suggested by ourselves and others $[1,19]$ that the disease could have a direct relationship to undernutrition during prenatal and early postnatal life. Although type 1 diabetes is strongly associated with low socioeconomic status and skeletal disproportion in this community [3], it remains unclear whether nutrition or related aspects of poverty influence disease development and contribute to the relatively late peak age of onset of their diabetes. Of note, communities with diverse ethnic backgrounds in sub-Saharan Africa, but without (reported) malnutrition, have found a later peak age 
incidence [2, 39]. One line of evidence does, however, point to the involvement of a complex gene-environment interaction affecting the age of disease onset. Ethiopian Jews moved in large numbers from rural areas around Gondar to urban areas of Israel in the 1980s and 1990s. After immigration to Israel the age of onset of type 1 diabetes in the young offspring of these Ethiopian emigres slowly fell (in those with at least two high-risk alleles for diabetes), in proportion to the time that their Ethiopian-born parents had been resident in Israel; at the same time the incidence of type 1 diabetes in these young people rose dramatically, to be one of the highest within the Jewish communities of Israel [44].

Another unexplained phenotypic feature in our study was the marked male preponderance in the post-pubertal but not pre-pubertal age groups; this is not a feature of type 2 diabetes in Ethiopia [4]. A less marked post-pubertal male preponderance in type 1 diabetes has been noted previously in European cohorts $[45,46]$. The cause of this striking sex bias is not clear, but may include sex-related immune and differential epigenome effects [17, 47].

\section{Limitations of the study}

There is wide genetic diversity in sub-Saharan Africa; that being the case, we have studied a single ethnic group to limit genetic diversity within this study and have emphasised that the results from the Semitic-background Amhara differ in autoantibody profile from the Bantu-background Cameroonians [2], who were investigated at a similar time, close to clinical diagnosis. Additionally, both the Amhara and Bantu have shown some differences from European background groups. Our results, therefore, are not fully generalisable, neither when confined to sub-Saharan Africa nor on a more global basis. However, the differences associated with genetic diversity can be used as a tool to help throw light on the relationships between genetic background and autoantibody profile in type 1 diabetes.

In summary, the majority of insulin-dependent diabetes in the Amhara of North-West Ethiopia is autoimmune in nature, and the genetic risk and protective factors for type 1 diabetes are largely common to those found in Europeans with type 1 diabetes. The results of this study and reports from other areas of sub-Saharan Africa highlight the need for a wider understanding of the gene-environment interactions giving rise to differences in timing of peak incidence, male preponderance (in those with post-pubertal onset) and autoantibody profile in type 1 diabetes.

Acknowledgements The authors thank C. Self, research student, for assistance with computing and M. McDonnell and A. Magill, Department of Clinical Biochemistry, Royal Victoria Hospital, Belfast for laboratory support. We are grateful to V. Lampasona, San Raffaele Scientific Institute, Milan and A. Lernmark, Lund University, Sweden for the plasmids used for islet autoantibody measurement.
Data availability The datasets generated during the current study are available by application to the corresponding author on reasonable request.

Funding DIWP and ERT received a grant from the Association of Physicians of Great Britain and Ireland; DLC is supported by a grant from the NIH, NIH1K99HD099330-01; SFAG is supported by the NIH (RO1 DK085212) and the Daniel B. Burke Endowed Chair for Diabetes Research; STJ and RDL are funded by Type 1 Diabetes UK (DUK 19/ 0005951); RDL is funded by the EFSD (MMBP1C3R), the EU (EUFP7:282510) and St Bartholomew's Hospital, London, Charity (OGA011582); TV is funded by the British Diabetic Twin Trust.

Authors' relationships and activities The authors declare that there are no relationships or activities that might bias, or be perceived to bias, their work.

Contribution statement $\mathrm{SAB}, \mathrm{AGD}, \mathrm{AG}$ and $\mathrm{AH}$ identified patients and control participants, were responsible for measuring and recording demographic data, and oversaw the procurement and initial storage of patient samples. RM, DLC, KMH, BFV, KL, SS and SFAG were responsible for designing, measuring and analysis of genetic assays. RM and DLC, BFV and $\mathrm{KL}$, were also involved in manuscript preparation. TV carried out data analysis. STJ performed genetic and data analysis. RDL was responsible for genetic design and analysis, and manuscript preparation. AJKW and HFW were responsible for autoantibody assay design and analysis. DIWP and ERT were responsible for conception, design, data collection, data analysis and manuscript preparation. All co-authors were involved in drafting and/or revising the article and have approved the final version of the manuscript. ERT is responsible for the integrity of the work as a whole.

Open Access This article is licensed under a Creative Commons Attribution 4.0 International License, which permits use, sharing, adaptation, distribution and reproduction in any medium or format, as long as you give appropriate credit to the original author(s) and the source, provide a link to the Creative Commons licence, and indicate if changes were made. The images or other third party material in this article are included in the article's Creative Commons licence, unless indicated otherwise in a credit line to the material. If material is not included in the article's Creative Commons licence and your intended use is not permitted by statutory regulation or exceeds the permitted use, you will need to obtain permission directly from the copyright holder. To view a copy of this licence, visit http://creativecommons.org/licenses/by/4.0/.

\section{References}

1. Balcha SA, Phillips DIW, Trimble ER (2018) Type 1 diabetes in a resource-poor setting: malnutrition related, malnutrition modified, or just diabetes? Curr Diab Rep 18:47. https://doi.org/10.1007/ s11892-018-1003-7

2. Asanghanwa M, Gorus FK, Weets I et al (2014) Clinical and biological characteristics of diabetic patients under age 40 in Cameroon: relation to autoantibody status and comparison with Belgian patients. Diabetes Res Clin Pract 103:97-105. https://doi. org/10.1016/j.diabres.2013.11.013

3. Fekadu S, Yigzaw M, Alemu S et al (2010) Insulin-requiring diabetes in Ethiopia: associations with poverty, early undernutrition and anthropometric disproportion. Eur J Clin Nutr 64:1192-1198. https://doi.org/10.1038/ejcn.2010.143 
4. Alemu S, Dessie A, Seid E et al (2009) Insulin-requiring diabetes in rural Ethiopia: should we reopen the case for malnutrition-related diabetes? Diabetologia 52:1842-1845. https://doi.org/10.1007/ s00125-009-1433-5

5. Panz VR, Kalk WJ, Zouvanis M, Joffe BI (2000) Distribution of autoantibodies to glutamic acid decarboxylase across the spectrum of diabetes mellitus seen in South Africa. Diabet Med 17:524-527. https://doi.org/10.1046/j.1464-5491.2000.00324.x

6. Lutale JJK, Thordarson H, Holm PI, Eide GE, Vetvik K (2007) Islet cell autoantibodies in African patients with type 1 and type 2 diabetes in Dar es Salaam Tanzania: a cross sectional study. J Autoimmune Dis 4:4. https://doi.org/10.1186/1740-2557-4-4

7. Siraj ES, Gupta MK, Yifter H et al (2016) Islet-cell associated autoantibodies in Ethiopians with diabetes mellitus. J Diabetes Complications 30:1039-1042. https://doi.org/10.1016/j.jdiacomp. 2016.05.005

8. Barrett JC, Clayton D, Concannon P et al (2009) Genome-wide association study and meta-analysis finds over 40 loci affect the risk of type 1 diabetes. Nat Genet 41:703-707. https://doi.org/10. 1038/ng.381

9. Campbell MC, Tishkoff SA (2008) African genetic diversity: implications for human demographic history, modern human origins, and complex disease mapping. Annu Rev Genomics Hum Genet 9:403-433. https://doi.org/10.1146/annurev.genom.9.081307. 164258

10. Gurdasani D, Carstensen T, Tekola-Ayele F et al (2015) The African Genome Variation Project shapes medical genetics in Africa. Nature 517:327-332. https://doi.org/10.1038/nature13997

11. Redondo MJ, Yu L, Hawa M et al (2001) Heterogeneity of type 1 diabetes: analysis of monozygotic twins in Great Britain and the United States. Diabetologia 44:354-362. https://doi.org/10.1007/ s001250051626

12. Op de Beeck A, Eizirik DL (2016) Viral infections in type 1 diabetes mellitus - why the $\beta$ cells? Nat Rev Endocrinol 12:263-273. https://doi.org/10.1038/nrendo.2016.30

13. Carlsson A, Kockum I, Lindblad B et al (2012) Low risk HLA-DQ and increased body mass index in newly diagnosed type 1 diabetes children in the Better Diabetes Diagnosis study in Sweden. Int J Obes 36:718-724. https://doi.org/10.1038/ijo.2011.122

14. Waterland RA, Lin JR, Smith CA, Jirtle RL (2006) Post-weaning diet affects genomic imprinting at the insulin-like growth factor 2 (Igf2) locus. Hum Mol Genet 15:705-716. https://doi.org/10.1093/ hmg/ddi484

15. Park JH, Stoffers DA, Nicholls RD, Simmons RA (2008) Development of type 2 diabetes following intrauterine growth retardation in rats is associated with progressive epigenetic silencing of $P d x 1$. J Clin Invest 118:2316-2324. https://doi.org/10.1172/ JCI33655

16. Sandovici I, Smith NH, Nitert MD et al (2011) Maternal diet and aging alter the epigenetic control of a promoter-enhancer interaction at the $H N F 4 \alpha$ gene in rat pancreatic islets. Proc Natl Acad Sci U S A 108:5449-5454

17. Moore SE, Cole TJ, Collinson AC, Poskitt EM, McGregor IA, Prentice AM (1999) Prenatal or early postnatal events predict infectious deaths in young adulthood in rural Africa. Int J Epidemiol 28: 1088-1095. https://doi.org/10.1093/ije/28.6.1088

18. Amare ZY, Endris M, Mehari AB (2019) Determinants of nutritional status among children under age 5 in Ethiopia: further analysis of the 2016 Ethiopia demographic and health survey. Glob Health 15:62. https://doi.org/10.1186/s12992-019-0505-7

19. Abdulkadir J, Mengesha B, Welde Gebriel Z et al (1990) The clinical and hormonal (C-peptide and glucagon) profile and liability to ketoacidosis during nutritional rehabilitation in Ethiopian patients with malnutrition-related diabetes mellitus. Diabetologia 33:222227
20. Alemu S, Dessie A, Tsegaw A et al (2015) Retinopathy in type 1 diabetes: major differences between rural and urban dwellers in northwest Ethiopia. Diabetes Res Clin Pract 109:191-198. https:// doi.org/10.1016/j.diabres.2015.04.010

21. Fort M, de Stefano G-F, Cambon-Thomsen A et al (1998) HLA class II allele and haplotype frequencies in Ethiopian Amhara and Oromo populations. Tissue Antigens 51:327-336. https://doi.org/ 10.1111/j.1399-0039.1998.tb02971.x

22. WHO Geneva (2009) WHO AnthroPlus software for personal computers. Available from www.who.int/growthref/tools/en/; accessed 15 Jun 2019

23. Kotler DP, Burastero S, Wang J, Pierson RN Jr (1996) Prediction of body cell mass, fat-free mass, and total body water with bioelectrical impedance analysis: effects of race, sex and disease. Am J Clin Nutr 64:489S-497S. https://doi.org/10.1093/ajen/64.3.489S

24. Bonifacio E, Yu L, Williams AK et al (2010) Harmonization of glutamic acid decarboxylase and islet antigen-2 autoantibody assays for National Institute of Diabetes and Digestive and Kidney Diseases consortia. J Clin Endocrinol Metab 95:3360 3367. https://doi.org/10.1210/jc.2010-0293

25. Long AE, Gillespie KM, Rokni S, Bingley PJ, Williams AJK (2012) Rising incidence of type 1 diabetes is associated with altered immunophenotype at diagnosis. Diabetes 61:683-686. https://doi. org/10.2337/db11-0962

26. Williams AJK, Lampasona V, Wyatt R et al (2015) Reactivity to Nterminally truncated $\operatorname{GAD}_{65}(96-585)$ identifies GAD autoantibodies that are more closely associated with diabetes progression in relatives of patients with type 1 diabetes. Diabetes 64:32473252. https://doi.org/10.2337/db14-1694

27. Purcell S, Neale B, Todd-Brown K et al (2007) PLINK: a tool set for whole-genome association and population-based linkage analyses. Am J Hum Genet 81:559-575. https://doi.org/10.1086/519795

28. Bunce M, O'Neill CM, Barnardo MC et al (1995) Phototyping: comprehensive DNA typing for HLA-A, B, C, DRB1, DRB3, DRB4, DRB5 \& DQB1 by PCR with 144 primer mixes utilizing sequence-specific primers (PCR-SSP). Tissue Antigens 46:355367. https://doi.org/10.1111/j.1399-0039.1995.tb03127.x

29. Zhou X, Stephens M (2012) Genome-wide efficient mixed-model analysis for association studies. Nat Genet 44:821-824. https://doi. org/10.1038/ng.2310

30. Mishra R, Chesi A, Cousminer DL et al (2017) Relative contribution of type 1 and type 2 diabetes loci to the genetic etiology of adult-onset, non-insulin-requiring autoimmune diabetes. BMC Med 15(1):88. https://doi.org/10.1186/s12916-017-0846-0

31. Mahajan A, Taliun D, Thurner M et al (2018) Fine-mapping of an expanded set of type 2 diabetes loci to single-variant resolution using high-density imputation and islet-specific epigenome maps. Nat Genet 50:1505-1513. https://doi.org/10.1038/s41588-0180241-6

32. Oram RA, Patel K, Hill A et al (2016) A type 1 diabetes genetic risk score can aid discrimination between type 1 and type 2 diabetes in young adults. Diabetes Care 39:337-344. https://doi.org/10.2337/ dc15-1111

33. Clarke L, Fairley S, Zheng-Bradley X et al (2017) The International Genome Sample Resource (IGSR): a worldwide collection of genome variation incorporating the 1000 Genomes project data. Nucleic Acids Res 45:D854-D859. https://doi.org/10.1093/nar/ gkw829

34. Wang J, Miao D, Babu S et al (2007) Prevalence of autoantibody negative diabetes is not rare at all ages and increases with older age and obesity. J Clin Endocrinol Metab 92:88-92. https://doi.org/10. 1210/jc.2006-1494

35. Kim CS, Song MK, Park JS et al (2007) The clinical and immunogenetic characteristics of adult-onset type 1 diabetes mellitus in Korea. Acta Diabetol 44:45-54. https://doi.org/10.1007/s00592007-0241-y 
36. Bravis V, Kaur A, Walkey HC et al (2018) Relationship between islet autoantibody status and the clinical characteristics of children and adults with incident type 1 diabetes in a UK cohort. BMJ Open 8:e020904. https://doi.org/10.1136/bmjopen-2017-020904

37. Gill GV, Tekle A, Reja A et al (2011) Immunological and Cpeptide studies of patients with diabetes in northern Ethiopia: existence of an unusual subgroup possibly related to malnutrition. Diabetologia 54:51-57. https://doi.org/10.1007/s00125-010-19217

38. Fan S, Kelly DE, Beltrame MH et al (2019) African evolutionary history inferred from whole genome sequence data of 44 indigenous African populations. Genome Biol 20:82. https://doi.org/10. 1186/s13059-019-1679-2

39. Padoa CJ, Rheeder P, Pirie FJ, Motala AA, van Dyk JC, Crowther NJ (2019) Identification of a subgroup of black South Africans with type 1 diabetes who are older at diagnosis but have lower levels of glutamic acid decarboxylase and islet antigen 2 autoantibodies. Diabet Med. https://doi.org/10.1111/dme.14204

40. Perry DJ, Wasserfall CH, Oram RA et al (2018) Application of a genetic risk score to racially diverse type 1 diabetes populations demonstrates the need for diversity in risk-modeling. Sci Rep 8: 4529. https://doi.org/10.1038/s41598-018-22574-5

41. Zhu M, Xu K, Chen Y et al (2019) Identification of novel T1D risk loci and their association with age and islet function at diagnosis in autoantibody-positive T1D individuals: based on a two-stage genome-wide association study. Diabetes Care 42:1414-1421. https://doi.org/10.2337/dc18-2023
42. Leslie RD, Grant SFA (2018) The dynamic origins of type 1 diabetes. Diabetes Care 41:2441-2443. https://doi.org/10.2337/dci180034

43. Battaglia M, Ahmed S, Anderson MS et al (2020) Introducing the endotype concept to address the challenge of disease heterogeneity in type 1 diabetes. Diabetes Care 43:5-12. https://doi.org/10.2337/ dc19-0880

44. Zung A, Elizur M, Weintrob $\mathrm{N}$ et al (2004) Type 1 diabetes in Jewish Ethiopian immigrants to Israel: HLA class II immunogenetics and contribution of new environment. Hum Immunol 65:14631468. https://doi.org/10.1016/j.humimm.2004.09.006

45. Pundziute-Lycka A, Dahlquist G, Nystrom L et al (2002) The incidence of type 1 diabetes has not increased but shifted to a younger age at diagnosis in the 0-34 years group in Sweden 1983-1998. Diabetologia 45:784-791

46. Kyvik KO, Nystrom L, Gorus F et al (2004) The epidemiology of type 1 diabetes mellitus is not the same in young adults as in children. Diabetologia 47:377-384. https://doi.org/10.1007/s00125004-1331-9

47. Khulan B, Cooper WN, Skinner BM et al (2012) Periconceptual maternal micronutrient supplementation is associated with widespread gender related changes in the epigenome: a study of a unique resource in the Gambia. Hum Mol Genet 21:2086-2101. https:// doi.org/10.1093/hmg/dds026

Publisher's note Springer Nature remains neutral with regard to jurisdictional claims in published maps and institutional affiliations.

\section{Affiliations}

\section{Shitaye A. Balcha ${ }^{1}$ • Abayneh G. Demisse ${ }^{2}$ - Rajashree Mishra ${ }^{3,4,5} \cdot$ Tanwi Vartak $^{6}$ - Diana L. Cousminer $3,5,7$. Kenyaita M. Hodge ${ }^{3,5} \cdot$ Benjamin F. Voight ${ }^{7,8} \cdot$ Kim Lorenz $^{7,8} \cdot$ Stanley Schwartz ${ }^{9} \cdot$ Samuel T. Jerram $^{6} \cdot$ Arla Gamper $^{10}$. Alice Holmes ${ }^{11} \cdot$ Hannah F. Wilson ${ }^{12}$ - Alistair J. K. Williams ${ }^{12}$ - Struan F. A. Grant ${ }^{3,5,7,13,14}$ • R. David Leslie ${ }^{6}$. David I. W. Phillips ${ }^{15} \cdot$ Elisabeth R. Trimble ${ }^{16}$}

1 Department of Internal Medicine, Gondar University Hospital, Gondar, Ethiopia

2 Department of Pediatrics and Child Health, School of Medicine, University of Gondar, Gondar, Ethiopia

3 Division of Human Genetics, The Children's Hospital of Philadelphia, Philadelphia, PA, USA

4 Graduate Group in Genomics and Computational Biology, Perelman School of Medicine, University of Pennsylvania, Philadelphia, PA, USA

5 Center for Spatial and Functional Genomics, The Children's Hospital of Philadelphia, Philadelphia, PA, USA

6 Blizard Institute, Queen Mary University of London, London, UK

7 Department of Genetics, Perelman School of Medicine, University of Pennsylvania, Philadelphia, PA, USA

8 Department of Systems Pharmacology and Translational Therapeutics, Perelman School of Medicine, University of Pennsylvania, Philadelphia, PA, USA
9 Main Line Health System, Wynnewood, PA, USA

10 Severn Postgraduate School of Primary Care, Health Education England, Bristol, UK

11 Avon and Wiltshire Mental Health Partnership NHS Trust, Clevedon, UK

12 Diabetes and Metabolism, Translational Health Sciences, University of Bristol, Southmead Hospital, Bristol, UK

13 Institute for Diabetes, Obesity and Metabolism, Perelman School of Medicine, University of Pennsylvania, Philadelphia, PA, USA

14 Department of Pediatrics, Perelman School of Medicine, University of Pennsylvania, Philadelphia, PA, USA

15 MRC Lifecourse Epidemiology Unit, University of Southampton, Southampton General Hospital, Southampton, UK

16 Centre for Public Health, Institute of Clinical Science, Queen's University Belfast, Grosvenor Road, Belfast BT12 6BA, UK 